\title{
Contribution to the Knowledge of Freshwater Halacarid Mites (Acari: Halacaridae) from Turkey
}

\author{
Furkan DURUCAN ${ }^{1 *}$ (D), Yunus Ömer BOYACI ${ }^{2}$ \\ ${ }^{1}$ Işıklar Caddesi No 16, 17 TR-07100 Antalya, Turkey \\ ${ }^{2}$ Isparta University of Applied Sciences, Faculty of Eğirdir Fisheries, Isparta, Turkey \\ *Corresponding Author: f_durucan@hotmail.com
}

Received 21 January 2019; Accepted 14 February 2019; Release date 15 September 2019.

How to Cite: Durucan, F., \& Boyac1, Y.Ö. (2019). Contribution to the knowledge of freshwater halacarid mites (Acari: Halacaridae) from Turkey. Acta Aquatica Turcica, 15(3), 318-324. https://doi.org/10.22392/actaquatr.515607

\begin{abstract}
Based on freshwater halacarid mites collected from four different provinces (Antalya, Denizli, Isparta and Muğla) of Turkey, two species of the genera Halacarellus and Porohalacarus are reported and illustrated in the present study. Halacarellus hyrcanus (Viets, 1928) was only recorded from Muğla, while Porohalacarus alpinus (Thor, 1910) is recorded from Isparta, Denizli and Antalya. H. hyrcanus is reported for the first time from Turkey in this study. Additionally, comparisons of the idiosoma lengths and localities of the two species are presented here.
\end{abstract}

Keywords: Halacaridae, Acari, Halacarellus hyrcanus, Porohalacarus alpinus.

Türkiye’nin Tatlısu Halacarid Kenelerine (Acari: Halacaridae) Katkılar

Özet

Bu çalışmada, Türkiye'nin 4 farklı ilinden (Antalya, Denizli, Isparta ve Muğla) toplanan örnekler üzerinden Halacarellus hyrcanus (Viets, 1928) ve Porohalacarus alpinus (Thor, 1910) türleri bildirilmiştir. H. hyrcanus sadece Muğla İli'nden kaydedilirken, $P$. alpinus türü Antalya, Denizli ve Isparta'dan kaydedilmiştir. H. hyrcanus türü Türkiye faunası için yeni kayıttır. Ayrıca, her iki türün vücut uzunlukları ve lokalite bilgileri de bu çalışmada sunulmuştur.

Anahtar kelimeler: Halacaridae, Acari, Halacarellus hyrcanus, Porohalacarus alpinus.

\section{INTRODUCTION}

Halacarid mites are relatively small benthic organisms, the adult body length is less than $1 \mathrm{~mm}$ and unable to swim. Although the majority are marine, some are found in freshwater streams, lakes and ponds. While the first marine halacarid mite recorded in 1758 by Baster, the first freshwater halacarid mite was described in 1879 by Kramer. Until now, the family Halacaridae includes more than 1000 marine and approximately 60 freshwater species all over the world (Bartsch, 2006, 2009; Durucan, 2018). In this study, illustrations of deutonymph and female of $P$. alpinus, and female, male of $H$. hyrcanus are given with their worldwide distributions and comparisons. The present materials from four different provinces (Antalya, Denizli, Isparta and Muğla) of Turkey adds new record of freshwater halacarid mites of Turkey.

\section{MATERIAL and METHODS}

Samples were collected by hand at localities in Turkey. Mites were extracted by washing the substrates. The meiofauna retained in the set of sieves $(63 \mu \mathrm{m}, 500 \mu \mathrm{m}, 1 \mathrm{~mm})$ was sorted under binocular microscope (Nikon SMZ 10A). In the laboratory, mite specimens were cleared in lactic acid and mounted in Hoyers medium. Figures were drawn with the aid of a camera lucida (Nikon Eclipse E400). The specimens were kept in the first author's personal collection in Antalya. All measurements are given as micrometers $(\mu \mathrm{m})$. 


\section{RESULTS}

Family Halacaridae Murray, 1877

Genus Halacarellus Viets, 1927

Halacarellus hyrcanus (Viets, 1928) (Figure 1)

\section{Material examined}

1q, 1 $^{\top}$, Karg1 Stream, Fethiye, Muğla, 15 May 2016, Coll. Y. Ö. Boyac1.

\section{Morphology and notes}

Dorsal and ventral plates large. Setae ds- 1 on AD, ds-2 to ds- 4 (striated in integument), ds- 5 on PD and ds- 6 on anal plate. AE with 3 pairs of ventral setae. PE with 1 dorsal seta and 2 ventral setae. Anterior portion of GA broadly rounded. Female GA with 2 pairs of pgs and sgs. Genital acetabula external (Figure 1). Male with almost 100 pgs around GO (Figure 1). Gnathosoma 1.6 times longer than wide. Both pairs of maxillary setae on rostrum. Palps 4 segmented. Total palp lenght is $150 \mu \mathrm{m}$. P-2 with dorsal seta; P-3 with medial spur; P-4 with three setae (Figure 1). Leg-I chaetotaxy (from trochanter to tarsus): 1, 2, 8, 6, 10, 8; Leg I longer than leg II (Figure 1). The morphological characteristics of the our specimens reported here accord with the previously descriptions (Bartsch 1998, 2018; Viets 1928). The present finding constitutes first record of this species from Turkey. See Table 1 for measurements.

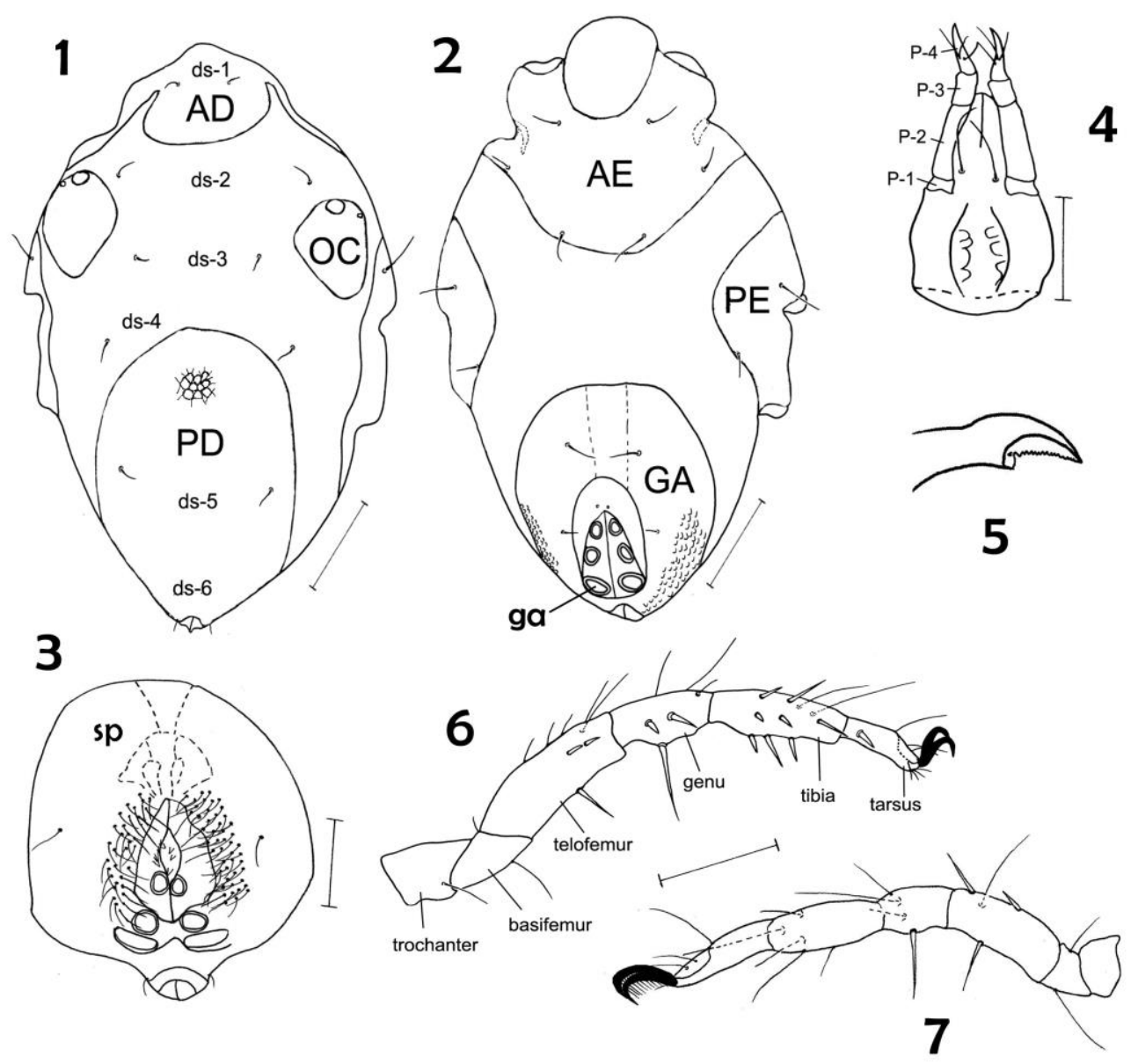

Figure 1. H. hyrcanus (Viets, 1928) 1 idiosoma, dorsal, $q 2$ idiosoma, ventral, $q \mathbf{3}$ GA, § 4 gnathosoma, ventral, $q \mathbf{5}$ chelicera, $q \mathbf{6}$ leg I, lateral, $q \mathbf{7}$ leg II, medial, $q$ (AD anterior dorsal plate AE anterior epimeral plate ds-1 to ds-6 dorsal setae numbered in sequence from anterior to posterior GA genitoanal plate ga genital acetabula OC ocular plate P-1 to P-4 palps from 1-4 PD posterior dorsal plate PE posterior epimeral plate sp spermatopositor) Scale bars: $100 \mu \mathrm{m}$. 


\section{Distribution}

H. hyrcanus was found for the first time from Caspian Sea, Bakü-Azerbaijan by Viets (1928) and afterwards recorded from Rhine (France, Germany, Netherlands), Bulgaria (Lake Varna), Romania (Danube delta, Sfintul Gheorghe, Plavisevita, Sulina) (Bartsch, 1998; 2004; 2009) Present record: Karg1 Stream, Fethiye (Muğla, Turkey) (Table 2).

Genus Porohalacarus Thor, 1922

Porohalacarus alpinus (Thor, 1910) (Figure 2-3)

\section{Material examined}

4 우, from Cladophora sp. on stone $50 \mathrm{~cm}$ depth (25 September 2016) (37 $53^{\prime} 0.5202 " \mathrm{~N}$; $\left.30^{\circ} 51^{\prime} 59.565^{\prime \prime} \mathrm{E}\right)$ at coastal part of Lake Eğirdir, Isparta; 6 우, from macrophytes which in 1-2 m depth (30 October 2014) (38 $\left.15^{\prime} 48.0^{\prime \prime} \mathrm{N} ; 29^{\circ} 55^{\prime} 17.0^{\prime \prime} \mathrm{E}\right)$ at Lake Işıklı, Denizli; 34 q $q$ and $3 \mathrm{DN}$, from macrophytes which in 30-50 cm depth (27 August 2018) (36 51'35.2872"N;3043'48.9468"E) at Mehmet Manavoğlu Park, Antalya. Coll. F. Durucan.

\section{Morphology and notes}

Dorsal and ventral plates large. Dark spots of eye pigment underneath $\mathrm{AD}$ and OC. The characteristics features of $P$. alpinus are dorsal plates reticulated, anterior margin of $\mathrm{AD}$ and $\mathrm{PD}$ truncated, female GA has five pairs of pgs; each of genital sclerit with four genital acetabula (Figure 1). The first author also observed four females with an everted ovopositor (Figure 1).

Gnathosoma 1.6 times longer than wide. Both pairs of maxillary setae on rostrum. Palps 4 segmented. Total palp lenght is $87 \mu \mathrm{m}$. P-2 with dorsal seta; P-3 with medial spur; P-4 with 3 setae (Figure 1). Leg-I chaetotaxy (from trochanter to tarsus): 1, 2, 4, 5, 6, 8. Legs slender, tibia I ventrally with four bristles (Figure 2). The same author also has found three deutonymphs at Mehmet Manavoğlu Park, Antalya. The deutonymph specimens similar to the adult one. Dorsal and ventral plates smaller than adults. GA of the specimens have four pairs of genital acetabula (Figure 3). See Table 1 for measurements.

P. alpinus differs from P. gallicus by the following features: (1) OC as long as or longer than AD in $P$. gallicus, while $\mathrm{OC}$ distinctively shorter than $\mathrm{AD}$ in $P$. alpinus (2) ventral plates fused to a ventral shield in P. gallicus while ventral plates are separated in P. alpinus (Bartsch, 2006).

The morphological characteristics and idiosoma size of our specimens accord with the original description. Table 2 summarizes worldwide literature data on idiosoma size of the species. This is the first halacarid record from Lake Iş1klı, Denizli, Turkey and constitutes the second record of this species from Sinop, Turkey, by Bartsch (2004) but the species not illustrated.

\section{Distribution}

P. alpinus was described (only one female) for the first time in Norway from littoral macrophytes by Thor (1910) and afterwards recorded from Europe (from Finland and Iceland to Italy), Black Sea (Turkey) North Africa, North America (United States and Canada), Australia and New Zealand (Bartsch, 2009); Madagascar (Bartsch, 2018) Present study records: Mehmet Manavoğlu Park (Antalya, Turkey), Lake Eğirdir (Isparta, Turkey) and Lake Iş̧1klı (Denizli, Turkey). 


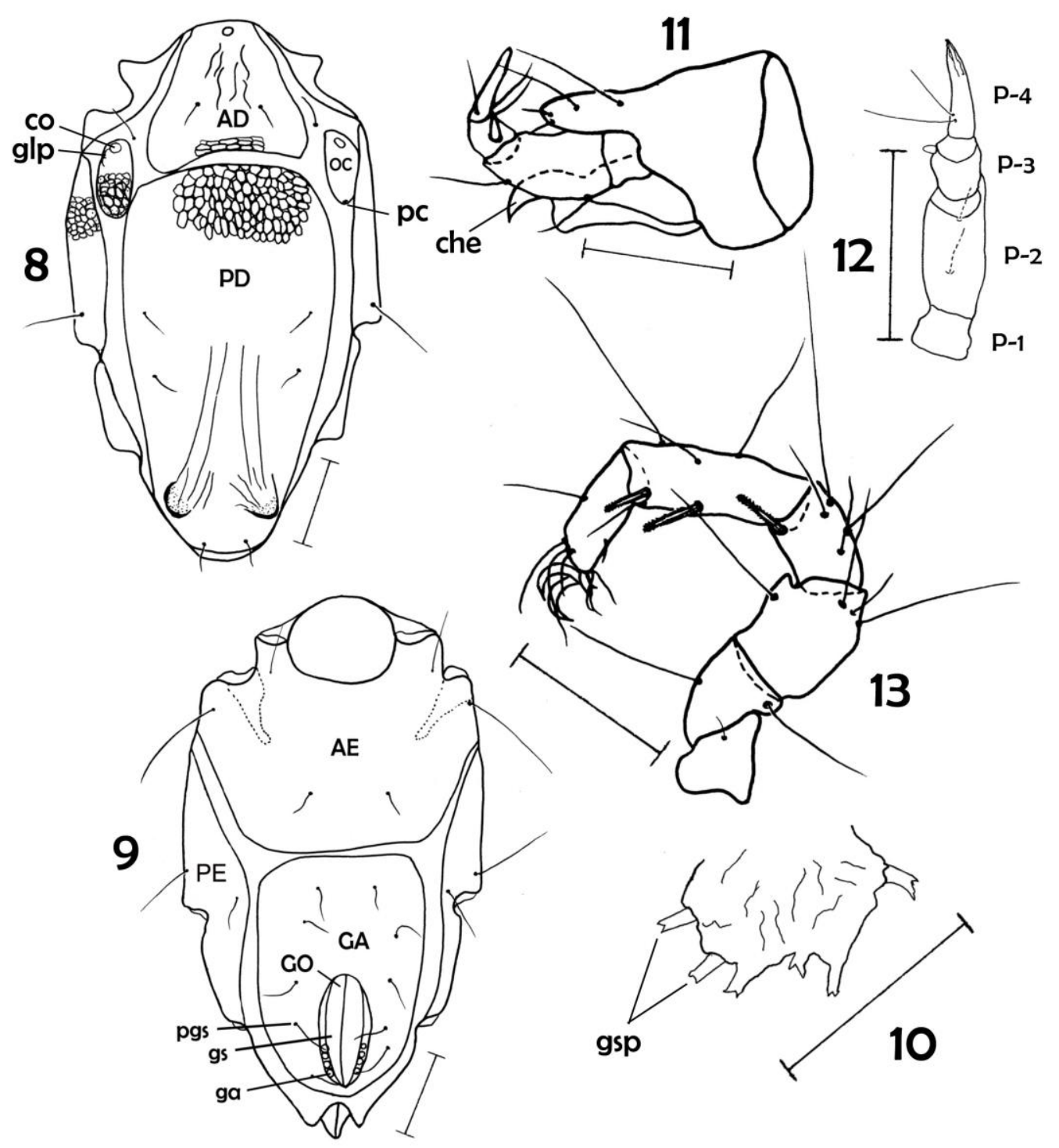

Figure 2. P. alpinus (Thor, 1910) female: 8 idiosoma, dorsal 9 idiosoma, ventral

10 everted ovopositor 11 gnathosoma, lateral 12 palp,ventro-lateral 13 leg I, lateral (AD anterior dorsal plate che chelicera co cornea AE anterior epimeral plate GA genitoanal plate ga genital acetabula glp gland pore

GO genital opening gs genital sclerite gsp genital spine OC ocular plate P-1 to P-4 palps from 1-4 pc pore canaliculus $\mathbf{P D}$ posterior dorsal plate $\mathbf{P E}$ posterior epimeral plate pgs perigenital setae) Scale bars: $50 \mu \mathrm{m}$. 


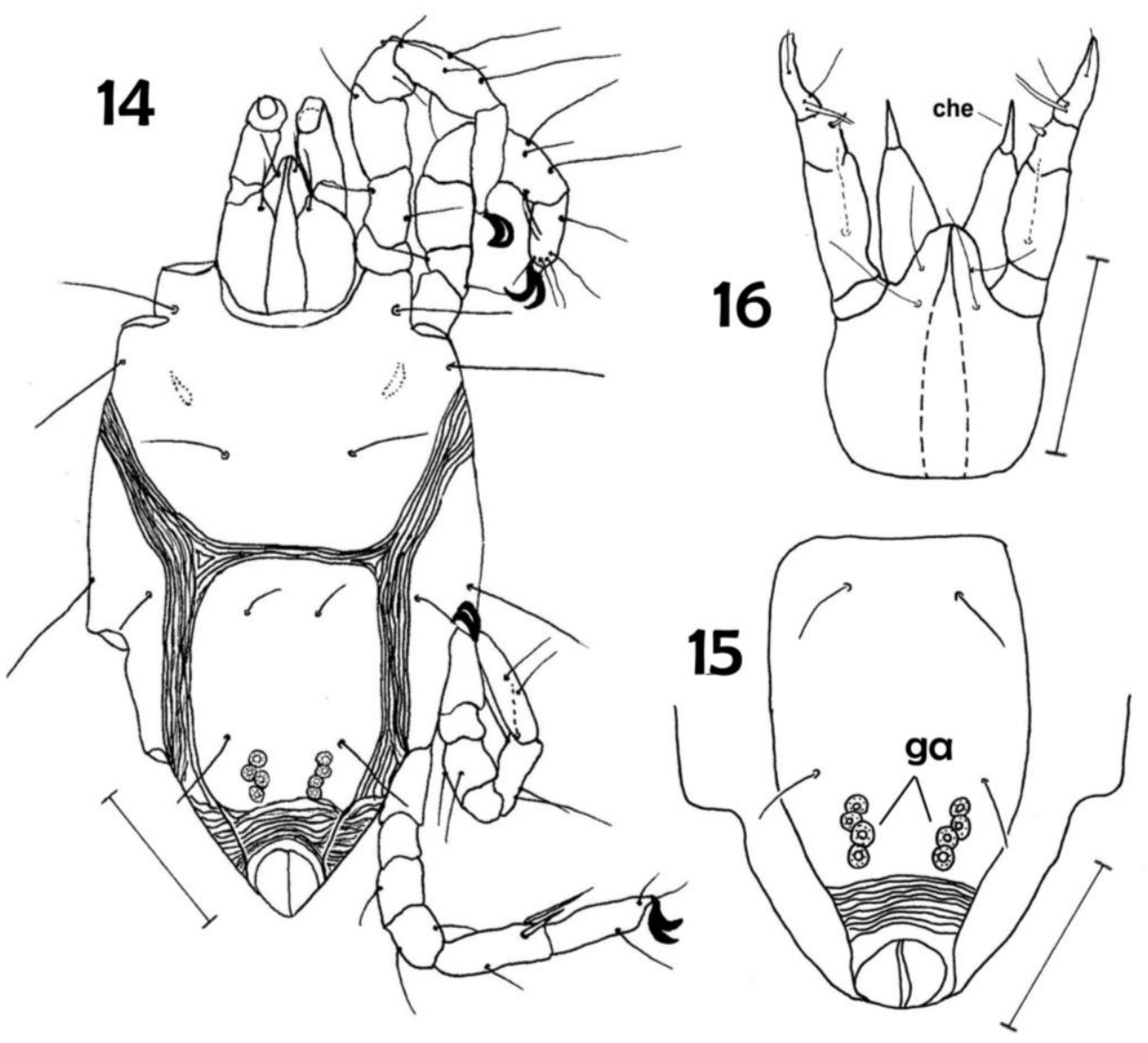

Figure 3. P. alpinus (Thor, 1910), DN: 14 Idiosoma with gnathosoma, ventral

15 deutonymph GA 16 gnathosoma, ventral (che chelicera ga genital acetabula) Scale bars: $50 \mu \mathrm{m}$.

Table 1. Measurements ( $\mu \mathrm{m})$ of $H$. hyrcanus and P. alpinus

\begin{tabular}{lllll}
\hline \hline \multirow{2}{*}{\begin{tabular}{l} 
Length/Width \\
\cline { 2 - 5 }
\end{tabular}} & \multicolumn{2}{c}{$\begin{array}{c}\text { Halacarellus hyrcanus } \\
\text { (Viets, 1928) }\end{array}$} & $\begin{array}{c}\text { Porohalacarus alpinus } \\
\text { (Thor, 1910) }\end{array}$ \\
\cline { 2 - 5 } Idiosoma & $+575 / 400$ & $550 / 375$ & $235-240 / 135-137$ & $+255-312 / 160-186$ \\
Gnathosoma & $200 / 110$ & $162 / 75$ & $55 / 50$ & $85 / 60$ \\
AD & $100 / 137$ & $125 / 170$ & $63 / 66$ & $75 / 100$ \\
OC & $100 / 75$ & $125 / 100$ & $37 / 25$ & $50 / 23$ \\
PD & $300 / 225$ & $300 / 250$ & $162 / 87$ & $225 / 125$ \\
AE & $137 / 287$ & $150 / 250$ & $75 / 125$ & $89 / 175$ \\
PE & $200 / 100$ & $250 / 100$ & $112 / 25$ & $162 / 37$ \\
GA & $237 / 225$ & $250 / 225$ & $77 / 65$ & $135 / 100$ \\
GO & $125 / 75$ & $110 / 90$ & - & $63 / 35$ \\
Total palp & 150 & 125 & 70 & 87 \\
Leg I & 550 & 412 & 155 & 225 \\
\hline \hline
\end{tabular}


Table 2. List of globally recorded $H$. hyrcanus and $P$. alpinus with additional informations (*, gnathosoma included $\mathbf{n}$, number of specimens $\mathbf{D N}$, deutonymph $\mathbf{L}$, larva $\mathbf{P N}$, protonymph)

\begin{tabular}{|c|c|c|c|}
\hline Species & Reference & Location & Length of idiosoma $(\mu \mathrm{m})$ \\
\hline \multirow{9}{*}{ P. alpinus } & Thor, 1910 & Norway & 우 $350 * / 200(n=1)$ \\
\hline & Walter, 1919 & Lake Davos-Switzerland & $?: 345 / 215(n=1)$ \\
\hline & Sokolov, 1952 & Russia & $+: 320 / 200$ \\
\hline & Bartsch, 1973 & Hohwachter Bucht-Germany & $\begin{array}{l}\text { O: } 343 / 265 \\
\text { DN: } 267-280 \\
\text { PN: } 205-209\end{array}$ \\
\hline & Morselli \& Mari, 1979 & $\begin{array}{l}\text { Parco della Repubblica, } \\
\text { Modena-Italy }\end{array}$ & $\begin{array}{l}\text { P: } 268-308 / 185-216(n=7) \\
\text { DN:162, 234, 276, } 318(n=4) \\
\text { PN:142, 208, 240, } 273(n=4)\end{array}$ \\
\hline & Mari \& Morselli, 1985 & Tuscan-Emilian Apennines-Italy & 우: 290-300/176-203 $(n=10)$ \\
\hline & Benfatti et al., 1992 & Lake Bolsena, Viterbo-Italy & ㅇ: 314/180, 315/174 (n=2) \\
\hline & Bartsch, 2018 & North and Southern Madagascar & $\begin{array}{l}\text { Ot: } 280-318(n=10) \\
\text { : } 286(n=1) \\
\text { DN: } 240-263(n=6) \\
\text { PN: } 186-210(n=6) \\
\text { L: } 140-185(n=6)\end{array}$ \\
\hline & Present study & $\begin{array}{l}\text { Lake Eğirdir, Isparta-Turkey } \\
\text { Lake Işıkl1, Denizli-Turkey } \\
\text { Mehmet Manavoğlu Park, } \\
\text { Antalya-Turkey }\end{array}$ & $\begin{array}{l}\text { O: } 250-287 / 162-175(\mathrm{n}=4) \\
\text { : } 255-312 / 160-186(\mathrm{n}=6) \\
\text { : } 250-300 / 135-188(\mathrm{n}=34) \\
\text { DN: } 210-232 / 130-135(\mathrm{n}=3)\end{array}$ \\
\hline \multirow{3}{*}{ H. hyrcanus } & Viets, 1928 & Caspian Sea, Bakü, Azerbaijan & 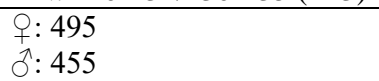 \\
\hline & Bartsch, 1998 & Lake Varna, Bulgaria & $\begin{array}{l}\text { o: } 478-527 \\
o: 447-459 \\
\text { DN: } 402-459\end{array}$ \\
\hline & Present study & $\begin{array}{l}\text { Karg1 Stream, Fethiye, Muğla- } \\
\text { Turkey }\end{array}$ & 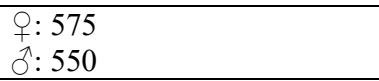 \\
\hline
\end{tabular}

\section{DISCUSSION}

In Turkey, the first records of freshwater halacarids (Copidognathus tectiporus and Porohalacarus alpinus) were given by Bartsch (2004). Later, Durucan (2018) recorded and illustrated all life stages of C. tectiporus from Lake Eğirdir (Isparta, Turkey).

$H$. hyrcanus is for the first time recorded from Turkey (Karg1 Stream, Muğla) in this study. Further studies aimed to improve our knowledge of Turkish freshwater halacarid mites should focus on unstudied areas and habitats in Turkey.

\section{REFERENCES}

Bartsch, I. (1973). Porohalacarus alpinus (Thor) (Halacaridae, Acari), ein morphologischer Vergleich mit marinen Halacariden nebst Bemerkungen zur Biologie dieser Art. Entomologisk Tidskrift, 74, 116-123.

Bartsch, I. (1998). Halacarinae (Acari, Halacaroidea) from the northwestern Black Sea: A review. Mitteilungen aus dem Hamburgischen Zoologischen Museum und Institut, 95, 143-178.

Bartsch, I. (2004). The Black Sea halacarid fauna (Halacaridae, Acari): faunal comparison with the Mediterranean, eastern North Atlantic, North Sea, and Baltic and reflection on its origin. Museum für Naturkunde in Berlin, Zoologische Reihe, 80, 2, 143-158.

Bartsch, I. (2006). 5. Acari: Halacaroidea. In: Gerecke R. (Ed.), Süßwasserfauna von Mitteleuropa 7/2-1, Chelicerata: Araneae, Acari I. Elsevier, Spektrum, Heidelberg, pp. 113-157.

Bartsch, I. (2009). Checklist of marine and freshwater halacarid mite genera and species (Halacaridae: Acari) with notes on synonyms, habitats, distribution and descriptions of the taxa, Zootaxa, 1998, 1-170.

Bartsch, I. (2018). Freshwater halacarid mites (Acari: Halacaridae) from Madagascar-new records, keys and notes on distribution and biology, Bonn zoological Bulletin, 67 (2), 79-99.

Benfatti, D., Mari, M., \& Morselli, I. (1992). Halacaroidea (Acari, Actinedida) from four lakes of volcanic origin in Lazio (Central Italy). Bollettino di Zoologia, 59, 105-111.

Durucan, F. (2018). First record of Copidognathus tectiporus Viets, 1935 (Halacaridae, Acari) from the Lake Eğirdir, Isparta, Turkey. Acta Biologica Turcica, 31 (1), 1-5. 
Mari, M., \& Morselli, I. (1985). Osservazioni su due Alacaridi (Acari, Prostigmata) dulciacquicoli raccolti in tre laghetti dell'Appennino. Tosco-Emiliano. Atti della Societa dei Naturalisti e Matematici di Modena, 116, 71-76.

Morselli, I., \& Mari, M. (1979). Sulla presenza di Porohalacarus alpinus (Thor) (Halacaridae, Acari) in un laghetto artificiale alla periferia di Modena. Bollettino del Museo Civico Storia Naturale di Verona, 6, 491-499.

Sokolov, I. I. (1952). Vodjanye klešči. II. Halacarae. Fauna SSSR, 5, 201 pp. Izdatelstvo Akademii NAUK SSSR, Moskova, Leningrad.

Thor, S. (1910). Die erste norwegische Süßwasserform der Halacariden. Zoologischer Anzeiger, 36, 348-351.

Viets, K. (1928). Wassermilben aus dem Schwarzen Meer, dem Kaspischen Meer und dem Aral-See. Abhandlungen Naturwissenschaftlicher Verein zu Bremen, 27, 47-80.

Walter, C. (1919). Schweizerische Süßwasserformen der Halacariden. Revue de Suisse Zoologie, 27, $235-242$. 